\title{
Improved outcomes on subcutaneous IgG in patients with humoral immunodeficiency and co-morbid bowel disease
}

\author{
Shaili N. Shah, Krista Todoric and Teresa K. Tarrant* \\ School of Medicine, University of North Carolina, USA
}

\begin{abstract}
Immunoglobulin replacement can be life-saving for certain individuals with immunodeficiencies. Subcutaneous immunoglobulin G (SCIG) is an increasingly used method of replacement over intravenous immunoglobulin G (IVIG), with potential advantages including fewer systemic side effects, no need for intravenous (IV) access, patient-reported improved quality of life, and decreased cost. However, while patients with certain associated co-morbidities, such as protein-losing enteropathy, may demonstrate more stable immunoglobulin G (IgG) levels when on SCIG compared to IVIG, the clinical significance of these experiences is not well described. Using retrospective chart review, we examined three cases in which SCIG and IVIG was administered to patients with either common variable immunodeficiency (CVID) or secondary humoral immunodeficiency and protein-losing gastrointestinal co-morbid disease. Both outpatient and inpatient records were reviewed for data regarding treatment with IVIG versus SCIG, reported frequency and severity of infections, hospitalizations, and IgG levels. All three patients demonstrated improvement in infection rate, stability of IgG levels, and co-morbid disease when on SCIG as compared to IVIG. These findings suggest that the pharmacokinetics of SCIG may translate into more consistent serum IgG levels, contributing to clinical improvement in immunodeficient patients with proteinlosing comorbidities when compared to IVIG. Limitations to this study are small patient numbers, retrospective design, and potential therapeutic bias. Further characterization of the effects of co-morbid conditions on immunoglobulin replacement is critical to providing improved and informed patient care.
\end{abstract}

\section{Background - Efficacy, safety, and pharmacokinetics of IgG replacement}

There are several options for immunoglobulin G (IgG) replacement, including multiple preparations of intravenous (IVIG) and subcutaneous (SCIG) IgG replacement. SCIG is becoming increasingly used with potential advantages including fewer systemic side effects, no need for IV access, improved patient-reported quality of life, and decreased cost [1].

A comparison study of the efficacy of IVIG versus SCIG in patients with primary antibody deficiency syndromes found no significant difference in efficacy as determined by number of infections [2]. Since then, patient preference and logistical factors have guided many providers' decision to treat with IVIG versus SCIG. Subsequently, a prospective study evaluating the safety of SCIG replacement found that the rate of systemic adverse reactions was low (approximately $1 \%$ and none classified as severe), and local cutaneous reactions declined over time [3]. In addition, investigators found that the mean IgG level increased from 7.8 to $9.2 \mathrm{~g} / \mathrm{L}$ in children, and from 8.6 to $8.9 \mathrm{~g} / \mathrm{L}$ in adults when individuals were switched from IVIG to SCIG therapy [3].

An important difference between SCIG and IVIG is the rapid peak of IgG level with IVIG when compared to a more steady state with SCIG [1]. IVIG is infused directly into the intravascular compartment while SCIG diffuses into the lymphatic compartment and enters the intravascular space via the thoracic duct, peaking at 48-72h [1]. Slower absorption seems to increase the systemic tolerability of the drug while the steady state maintained with SCIG has been suggested to be more physiologic, similar to IgG levels maintained in healthy patients.

As our knowledge regarding primary immunodeficiency diseases expands, emerging are scenarios in which SCIG therapy may be advantageous. A case report looking at SCIG in a child with primary intestinal lymphangiectasia and secondary hypogammaglobulinemia found that weekly administration of SCIG allowed for a more steady state of serum IgG than monthly IV bolus dosing [4]. An early study examining IgG bioavailability after subcutaneous infusion in common variable immune deficiency (CVID) patients found that the serum IgG concentration increased significantly in patients previously treated with intramuscular or intravenous IgG [5]. Later studies have supported this, showing that, at equivalent total monthly dose, weekly SCIG results in steady state levels $10-20 \%$ higher than troughs on monthly IVIG [6]. Additionally, a cohort study showed that CVID patients with disease related conditions (enteropathy, cytopenias, lymphoid proliferation) had significantly lower IgG on IVIG compared to those on SCIG [7]. This outcome did not achieve statistical significance when each comorbidity was independently analyzed, and comparative responses of SCIG versus IVIG replacement in the same patient were not discussed. As a result of the aforementioned data, we hypothesized that SCIG would be a superior method of IgG replacement in CVID patients with protein-losing bowel disease, and the following cases highlight

Correspondence to: Teresa K. Tarrant, MD, Associate Professor of Medicine and Microbiology and Immunology, University of North Carolina School of Medicine, Division of Rheumatology, Allergy and Immunology, Thurston Arthritis Research Center and Lineberger Comprehensive Cancer Center, 3300 Manning Drive CB \#7280, Chapel Hill, NC 27599, Tel: (919) 843-4727, E-mail: Tarra002@med.unc.edu

Key words: IgG replacement therapy, subcutaneous, primary immune deficiency, common variable immunodeficiency (CVID), protein-losing enteropathy, inflammatory bowel disease (IBD)

Received: June 21, 2015; Accepted: July 25, 2015; Published: July 28, 2015 
individual clinical presentations in which SCIG proved to be a better therapy than IVIG.

\section{Patient 1}

Patient 1 presented to Immunology at age 44 when she was hospitalized for pneumonia. She had a history of numerous bacterial pneumonias starting in childhood, and laboratory evaluation in her twenties showed poor vaccination response to rubella, mildly decreased IgG at $600 \mathrm{mg} / \mathrm{dL}$, and absolute IgA deficiency. A few years later, she was formally diagnosed with CVID when her IgG dropped to $240 \mathrm{mg} / \mathrm{dL}$ with persistently undetectable IgA, and she was started on IVIG replacement. Upon presentation to our group, she was on an IVIG dose of $280 \mathrm{mg} / \mathrm{kg}$ with an IgG trough of $270 \mathrm{mg} / \mathrm{dL}$ despite having been previously therapeutic for over 10 years, and she reported having developed new onset diarrhea and weight loss. Her IVIG dose was titrated to $686 \mathrm{mg} / \mathrm{kg}$ with IgG troughs remaining low in the $300 \mathrm{~s}$. She was then switched to $940 \mathrm{mg} / \mathrm{kg}$ SCIG with subsequent IgG levels ranging 500-917 $\mathrm{mg} / \mathrm{dL}$, even prior to starting immunosuppression for her inflammatory bowel disease. Since then, she has not been hospitalized with any severe infections. She continues to have 1-2 episodes of sinusitis/year treated with oral antibiotics. Since developing diarrhea, she has been diagnosed with CVID associated inflammatory bowel disease with biopsy showing small bowel granulomata, villous blunting, and increased intraepithelial lymphocytes and is now on immunosuppression with mycophenolate mofetil 1-2 grams daily with adequate control of her bowel symptoms.

\section{Patient 2}

Patient 2 presented to Immunology at age 62 when she was admitted for chronic diarrhea and weight loss. She had a history of chronic lymphocytic leukemia in remission and post-chemotherapy pan-hypogammaglobulinemia (IgG $205 \mathrm{mg} / \mathrm{dL}$, IgM $125 \mathrm{mg} / \mathrm{dL}$, IgA undetectable) for which she was receiving monthly IVIG. While on an unknown dose of IVIG at an outside institution, she had several admissions for pneumonia and chronic sinusitis requiring IV antibiotic therapy and was transitioned to SCIG. After transition to SCIG, she had weight gain and decreased diarrhea. Her IgG levels ranged 650$705 \mathrm{mg} / \mathrm{dL}$ while on approximately $780 \mathrm{mg} / \mathrm{kg}$ of SCIG. She required one course of outpatient antibiotic therapy for sinusitis in the following two years. Her chronic diarrhea was diagnosed as immune deficiencyassociated enteropathy, and biopsy showed partial villous atrophy of the duodenum and increased intraepithelial lymphocytes.

\section{Patient 3}

Patient 3 presented to the Immunology clinic at age 42 with a few years history of recurrent infection, thrombocytopenia, and granulomatous lung lesions. She developed recurrent pneumonia leading to five hospitalizations in two years. She also suffered from diarrhea for $>10$ years with biopsy showing inflammatory disease with ileitis and colitis. Laboratory data revealed hypogammaglobulinemia (IgG $329 \mathrm{mg} / \mathrm{dL}$, IgA $15 \mathrm{mg} / \mathrm{dL}$ ) and absent responses to tetanus and pneumococcal vaccination. She received an initial dose of IVIG with immediate transition to SCIG. While on approximately $270 \mathrm{mg} / \mathrm{kg}$ of SCIG, she maintained her IgG level $>1000 \mathrm{mg} / \mathrm{dL}$ and had significant improvement in her infection frequency and severity. Her bowel disease waxed and waned, requiring immunosuppressive therapy. In contrast to the preceding cases, this patient was placed on SCIG early on in her disease course and did very well despite multiple co-morbid conditions that could potentially interfere with IgG metabolism and maintenance of therapeutic levels. Because of her early transition to SCIG, we cannot definitively conclude that SCIG was superior to IVIG and only that her clinical course was benefitted by the early addition of SCIG replacement.

\section{Discussion}

The humoral immune deficient patients with co-morbid bowel disease in our case series have shown clinical and serologic improvement while on SCIG replacement therapy. This suggests that the pharmacokinetic studies performed in the past translate into clinical practice and that there is more to be explored with regards to the effects of co-morbid conditions on the clearance of IgG. Limitations of this study include small patient sample, retrospective design, and potential therapeutic bias. In addition, the conversion from IVIG to SCIG was not $1: 1$, given the retrospective nature of this review and the initial dosing recommendations that SCIG be given at $137 \%$ of the IVIG dose [8]. Concurrent treatment of underlying enteropathy may also play a role in improvement of IgG levels in some cases. The final decision of using SCIG versus IVIG remains based on an important discussion between the patient and physician, considering multiple factors not limited to cost, patient preference, convenience, compliance, and comorbid disease. Further characterization of the effects of co-morbid conditions on IgG replacement will be critical to providing improved and informed patient care.

\section{Conflict of interest}

The authors have no conflicts of interest to disclose.

\section{References}

1. Berger M (2004) Subcutaneous immunoglobulin replacement in primary immunodeficiencies. Clin Immunol 112: 1-7. [Crossref]

2. Chapel HM, Spickett GP, Ericson D, Engl W, Eibl MM, et al. (2000) The comparison of the efficacy and safety of intravenous versus subcutaneous immunoglobulin replacement therapy. J Clin Immunol 20: 94-100. [Crossref]

3. Gardulf A, Nicolay U, Asensio O, Bernatowska E, Bock A, et al. (2006) Rapid subcutaneous IgG replacement therapy is effective and safe in children and adults with primary immunodeficiencies--a prospective, multi-national study. J Clin Immunol 26: 177-85. [Crossref]

4. Lin JH, Roberts RL (2003) Subcutaneous immunoglobulin infusions for treatmen of a child with severe protein-losing enteropathy. J Allergy Clin Immunol 113(2, Supplement): S42.

5. Waniewski J, Gardulf A, Hammarstrom L (1994) Bioavailability of gamma-globulin after subcutaneous infusions in patients with common variable immunodeficiency. $J$ Clin Immunol 14: 90-7. [Crossref]

6. Berger M (2011) Choices in IgG replacement therapy for primary immune deficiency diseases: subcutaneous IgG vs. intravenous IgG and selecting an optimal dose. Curr Opin Allergy Clin Immunol 11: 532-8. [Crossref]

7. Gouilleux-Gruart V, Chapel H, Chevret S, Lucas M, Malphettes M, et al. (2013) Efficiency of immunoglobulin $\mathrm{G}$ replacement therapy in common variable immunodeficiency: correlations with clinical phenotype and polymorphism of the neonatal Fc receptor. Clin Exp Immunol 171: 186-194. [Crossref]

8. Ochs HD, Gupta S, Kiessling P, Nicolay U, Berger M, et al. (2006) Safety and efficacy of self-administered subcutaneous immunoglobulin in patients with primary immunodeficiency diseases. J Clin Immunol 26: 265-73. [Crossref]

Copyright: $(2015$ Onuigbo W. This is an open-access article distributed under the terms of the Creative Commons Attribution License, which permits unrestricted use, distribution, and reproduction in any medium, provided the original author and source are credited. 